\title{
PERTURBATIONS HÉPATIQUES INDUITES EXPÉRIMENTALEMENT PAR UNE CONCENTRATION SUBLÉTALE DE PROCYMIDONE (FONGICIDE DICARBOXIMIDE) CHEZ BRACHYDANIO RERIO (TÉLÉOSTÉEN, CYPRINIDÉ) : APPROCHE HISTO-CYTOPATHOLOGIQUE.
}

\author{
PARIS-PALACIOS S., BIAGIANTI-RISBOURG S. et VERNET G. \\ Laboratoire de Zoologie et des Sciences de l'Environnement, \\ E.A. 2069 U.F.R. Sciences Exactes et Naturelles, \\ Bâtiment Europol'Agro, \\ B.P. 1039, 51687 Reims Cedex 2, France.
}

\section{RÉSUMÉ}

La procymidone[ $N$-(3,5 dichlorophényl)-1,2 diméthylcyclopropane-1,2 dicarboximide] est une molécule utilisée pour ses capacités antifongiques principalement dans la lutte contre Botrytis et Sclerotinia. Elle présente une rémanence de plusieurs semaines dans l'environnement et est donc susceptible de contaminer, après lessivage des sols, les milieux aquatiques et les organismes qui les peuplent.

L'analyse des perturbations histo-cytologiques hépatiques induites chez le Cyprinidé Brachydanio rerio par une concentration sublétale $\left(1 \mathrm{mg} . \mathrm{I}^{-1}\right)$ de ce fongicide a été réalisée après 4,7 et 14 jours de contamination expérimentale. L'éventuelle réversibilité des perturbations induites a été étudiée après 14 jours de contamination suivis de 14 jours de décontamination.

Les résultats révèlent des réponses hépatocytaires non spécifiques à l'agression toxique et d'intensité croissante en fonction de la durée de contamination. II s'agit de réponses adaptatives (déplétion glycogénique, accumulation lipidique, réduction de la surface d'absorption hépatocellulaire...) et de réponses dégénératives (désorganisation des travées hépatocytaires, lyse du parenchyme dans les régions périvasculaires...). Ces réponses s'accompagnent d'une activation notable de la réponse immunitaire cellulaire non spécifique (augmentation du nombre des macrophages) en relation avec l'accroissement du catabolisme tissulaire hépatique induit. Des perturbations plus originales ont été observées : il s'agit de développements d'une organisation concentrique des travées hépatocytaires et de protrusions cytoplasmiques entre hépatocytes adjacents amenant parfois la membrane plasmique au contact de l'enveloppe nucléaire. Le retour en eau non contaminée provoque la réduction des atteintes hépatiques (réversibilités des perturbations induites) et une forte activation du métabolisme hépatocytaire (hyperdéveloppement du réticulum endoplasmique, accroissement du nombre des mitochondries).

\section{HEPATIC DISORDERS INDUCED BY A SUBLETHAL CONCENTRATION OF PROCYMIDONE (FONGICIDE DICARBOXIMIDE) IN BRACHYDANIO RERIO (TELEOSTEI, CYPRINIDAE) : HISTO-CYTOLOGICAL APPROACH.}

\begin{abstract}
Procymidone [ $\mathrm{N}-(3,5$ dichlorophenyl)-1,2 dimethylcyclopropane-1,2 dicarboximide] is a fungicide frequently used against Botrytis, Sclerotinia and others. It persists several weeks in soils and thus may pollute aquatic environments and organisms. Non-specific liver histo-cytological disorders in Brachydanio rerio exposed to a sublethal concentration of $1 \mathrm{mg.l^{-1 }}$ of the fungicide occurred after 4,7 and 14 days of exposure. They were timedependent. Adaptative (glycogen depletion, lipid accumulation, disappearance of the
\end{abstract}


perisinusoidal microvillies) and degenerative events (disorganization of hepatic cords, perivascular lysis of the parenchyma) associated with an increase of the non-specific cellular immune response (increased number of macrophages) occurred. Additionally, unusual perturbations were observed : development of concentric hepatocyte arrangements and of cytoplasmic protrusions between adjacent hepatocytes. Hepatic recovery was obvious after 14 days of depuration (reduction of the lytic features and metabolic activation of hepatocytes).

The results give evidence of microscopic disorders in $B$. rerio liver induced by procymidone. The analysis of liver responses in experimentally exposed fish can provide a basis for recognizing toxicity syndromes and may therefore be used as biomarker in the prognosis of the effects of aquatic pollutants.

\section{INTRODUCTION}

L'utilisation de pesticides rémanents en agriculture provoque, après lessivage des sols, une pollution chronique plus ou moins sévère des milieux aquatiques. Parmi ceux-ci, citons la procymidone [ $\mathrm{N}$-(3,5 dichlorophenyl)-1,2 diméthylcyclopropane-1,2dicarboximide], un fongicide largement utilisé pour le traitement de tous types de cultures : fruits, vignes, légumes, céréales, fleurs... Elle est stable à la lumière, à la chaleur et à l'humidité et elle persiste 3 mois dans l'environnement. Chez les Poissons, ce dicarboximide présente une toxicité aiguë variable en fonction des espèces avec une $96 \mathrm{~h}-\mathrm{CL} 50$ de $22,9 \mathrm{mg}^{-1}{ }^{-1}$ chez la perche soleil Lepomis macrochirus et de $3,6 \mathrm{mg} .1^{-1}$ chez la truite arc-en-ciel Oncorhynchus mykiss (Pesticide manual, 1997).

Les études consacrées à l'impact biologique de la procymidone chez les Vertébrés aquatiques sont très rares et se limitent à des tests de toxicité aiguë. Dans le but d'analyser la toxicité sublétale de ce pesticide, les perturbations histo-cytopathologiques du foie induites par $1 \mathrm{mg} . \mathrm{I}^{-1}$ de procymidone pure ont été étudiées chez Brachydanio rerio dont la biologie est bien connue (LAALE, 1977). Ce Cyprinidé est un organisme normalisé (AFNOR, 1985), fréquemment utilisé (BRESCH, 1993 ; NAGEL, 1993 ; PHROMKUTHONG et al., 1994) et recommandé par l'OCDE pour les études de toxicologie de l'environnement. Le foie est un organe clé intervenant à différents niveaux de la physiologie des Poissons, en particulier dans les processus de détoxication de certains pesticides lipophiles [induction de monooxygénases cytochrome-P450 dépendantes (MFO) essentiellement associées au réticulum endoplasmique : OULMI et al., 1995]. De plus, l'hépatocyte constitue un modèle sensible souvent utilisé en toxicologie de l'environnement. Certaines de ses réponses (ultra)structurales ont, par ailleurs, été déterminées comme biomarqueurs de pollution (revues de HINTON et LAUREN, 1990 ; BRAUNBECK, 1995 et BIAGIANTI-RISBOURG, 1997). De plus, des données récentes de la littérature montrent une corrélation entre l'importance des atteintes dégénératives du foie et la capacité de survie des Poissons (BIAGIANTI-RISBOURG et al., 1996).

L'éventuelle réversibilité des atteintes hépatiques induites par la procymidone, permettant d'analyser la sévérité des perturbations, a été étudiée après retour des poissons en eau non contaminée.

\section{MATÉRIELS ET MÉTHODES}

Provenant d'un élevage commercial, 60 danios (P.T.: $0,20 \mathrm{~g}$, D.S.: $0,07 \mathrm{~g}$ et L.T.: $3,24 \mathrm{~cm}$, D.S.: $0,52 \mathrm{~cm}$ ) ont été acclimatés 3 semaines aux conditions d'élevage. Les femelles en vitellogenèse (50 individus dont l'état de maturation gonadique a été contrôlé en histologie) ont ensuite été réparties dans des aquariums témoins ou contaminés par une concentration sublétale de $1 \mathrm{mg}^{-1}$ de procymidone pure ( $D^{\prime}$ EHRENSTORFER $\mathrm{GmbH}$, Augsburg). Le fongicide a été dilué dans une eau de source provenant de la région Champagne-Ardenne (eau "Cristaline", Source des Grands Bois, Fismes) et contenant $124 \mathrm{mg} . \mathrm{l}^{-1} \mathrm{Ca}^{2+}, 25 \mathrm{mg} . \mathrm{l}^{-1} \mathrm{Mg}^{2+}, 11 \mathrm{mg.l} \mathrm{l}^{-1} \mathrm{Na}^{2+}, 3,5 \mathrm{mg} . \mathrm{l}^{-1} \mathrm{~K}^{+}, 420 \mathrm{mg}^{-1} \mathrm{CaCO}^{3-}$, $16 \mathrm{mg} . \mathrm{I}^{-1} \mathrm{Cl}^{-}$et $60 \mathrm{mg} . \mathrm{l}^{-1} \mathrm{SO}_{4}^{2-}$. Les élevages expérimentaux ont été menés en triplicatas 
avec une densité de 4 poissons $\mathrm{I}^{-1}$. Les poissons ont été nourris journellement, ad libitum, avec un aliment artificiel (TetraMin). La température a été maintenue à $20 \pm 1^{\circ} \mathrm{C}$ et le pH à $8 \pm 0,5$. La photophase et la scotophase étaient de $12 \mathrm{~h}$. La concentration de l'eau en procymidone a été maintenue stable pendant toute la durée des expériences (tests semistatiques avec renouvellement de l'eau tous les 2 jours) et contrôlée par dosage en HPLC.

Après $4,7,14$ jours de contamination et 14 jours de contamination suivis de 14 jours de décontamination, chaque prélèvement a concerné 6 femelles intoxiquées et 3 témoins. Pour les dosages de procymidone hépatique, des pools de foies $(0,03 \pm$ $0,001 \mathrm{~g}$ ) de 3 femelles intoxiquées ou de 3 témoins ont été broyés dans de l'acétonitrile à $4^{\circ} \mathrm{C}$, centrifugés $10 \mathrm{~min}$. à $4000 \mathrm{tr}_{\mathrm{min}}^{-1}$ et le pesticide contenu dans le surnageant a été dosé par HPLC.

\section{Dosage de la procymidone}

La séparation a été effectuée à l'aide d'une colonne Spherisorb (S5.ODS.2) de $25 \mathrm{~cm} \times 4,6 \mathrm{~mm}$ en phase inverse grâce à un gradient à haute pression de tampon phosphate/acétonitrile : $40 / 60$ sous un débit de $1 \mathrm{ml}^{\mathrm{min}}{ }^{-1}$. La détection a été réalisée par absorption U.V. à barrette de diodes (Ginkotek UVD340S).

\section{Histologie}

Les échantillons ont été fixés au Bouin Hollande, déshydratés et inclus dans de la paraffine. Les coupes ( $5 \mu \mathrm{m}$ d'épaisseur) ont été colorées au rouge nucléaire solide et picro-indigocarmin (RNS-PIC) ; un contrôle histochimique de la charge glycogénique hépatique a été réalisé par coloration à l'aide de l'acide périodique-réactif de Schiff (APS) avec (individus intoxiqués) ou sans (témoins) coloration nucléaire à l'hématoxiline de Groat (MARTOJA Et MARTOJA-PIERSON, 1967).

\section{Cytologie}

Des pièces de foie de petites tailles $\left(1 \mathrm{~mm}^{3}\right)$ ont été fixées par immersion (3h à $\left.4^{\circ} \mathrm{C}\right)$ dans du glutaraldéhyde à $3 \%$ dilué dans le mélange cacodylate de sodium $(0,1 \mathrm{M})$ chlorure de calcium $(0,5 \%)$, rincées dans ce tampon puis post-fixées ( $1 \mathrm{~h}$ à $4^{\circ} \mathrm{C}$ ) par du tétroxyde d'osmium à $1 \%$ dans le tampon contenant du saccharose $(0,3 \mathrm{M})$. Ces échantillons ont ensuite été inclus dans du spurr. Des coupes histologiques semi-fines (1 $\mu \mathrm{m}$ d'épaisseur) ont été colorées au bleu de méthylène-azur II (BMAII) selon RICHARDSON et al. (1960). Les coupes fines (0,05 $\mu \mathrm{m}$ d'épaisseur) contrastées à l'acétate d'uranil et au citrate de plomb selon REYNOLDS (1963) ont ensuite été observées à l'aide d'un microscope électronique à transmission (Philips EM 201, CIME Jussieu Paris).

\section{RÉSULTATS}

Les poissons contaminés n'ont pas présenté de perturbations comportementales et aucune mortalité n'a été observée au cours des 28 jours d'expérimentation.

\section{Concentration hépatique de la procymidone}

Les dosages effectués (Tableau I) montrent que les individus exposés au fongicide présentent des facteurs de bioaccumulation (FB) relativement importants. Au $4^{e}$ jour d'exposition, ce pesticide est fortement accumulé dans le foie. Après 7 jours et jusqu'à la fin de la période de contamination, le FB se stabilise à une valeur nettement plus faible. $\mathrm{Au}$ cours du retour en eau non contaminée, la bioconcentration en fongicide diminue notablement. 


\section{Tableau I}

Facteur de bioaccumulation (FB) de la procymidone dans le foie des danios au cours de la contamination et de la décontamination (n.d. = non détecté). La concentration de procymidone dans l'eau étant de $1 \mathrm{mg.l^{-1 }}$, le FB est égal à la concentration en procymidone du foie $\left(\mathrm{mg} \cdot \mathrm{kg}^{-1}\right)$.

\section{Table I}

Bioaccumulation factor (FB) of procymidone in liver during exposure and decontamination periods $($ n.d. $=$ not detected). Since the water concentration of procymidone was $1 \mathrm{mg} \cdot \mathrm{I}^{-1}$, the FB is the [procymidone] of the liver ( $\mathrm{mg}^{\mathrm{kg}} \mathbf{k g}^{-1}$ ).

\begin{tabular}{|c|c|c|c|c|c|}
\cline { 2 - 5 } \multicolumn{1}{c|}{} & \multirow{2}{*}{ Témoins } & \multicolumn{3}{|c|}{ Contamination } & Contamination $14 \mathrm{j} .+$ \\
\cline { 2 - 5 } & & $4 \mathrm{j}$. & $7 \mathrm{j}$. & $14 \mathrm{j}$. & Décontamination $14 \mathrm{j}$. \\
\hline F.B. & n.d. & $1150 \pm 9$ & $140 \pm 5$ & $160 \pm 6$ & $40 \pm 3$ \\
\hline
\end{tabular}

\section{(Ultra)structure du foie des témoins}

Le foie des femelles en vitellogenèse (Fig. 1) présente un parenchyme homogène composé d'hépatocytes organisés en travées bicellulaires séparées par des sinusoïdes. Ces sinusoïdes sont de fins capillaires sanguins limités par un endothelium fenestré qui drainent le sang des afférences portales et le distribuent aux afférences sus-hépatiques (Fig. 1). Quelques canaux biliaires sont bien visibles dans le parenchyme.
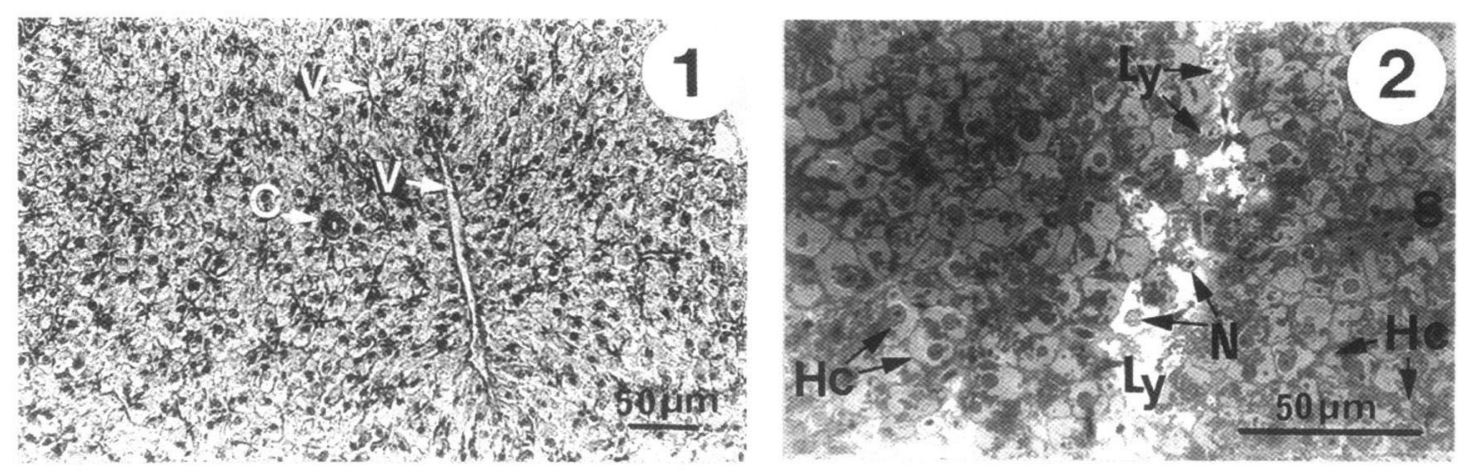

Figure 1

Parenchyme hépatique homogène des danios témoins. $\mathrm{V}=$ veine, $\mathrm{C}=$ canal biliaire. Coloration RNS-PIC.

Figure 1

Homogenous structure of hepatic parenchyma from danio controls. $V=$ vein, $\mathrm{C}=$ biliary canal. Coloration RNS-PIC (nuclear fast red-picro indigocarmin).

\section{Figure 2}

Après $7 \mathrm{j}$. de contamination, le parenchyme hépatique montre d'importantes plages de lyse (Ly). Hc = hépatocyte clair, $\mathrm{N}=$ noyau. Coloration BMAll.

\section{Figure 2}

After 7 days of exposure, the hepatic parenchyma displayed large lysis areas (Ly). $\mathrm{Hc}=$ clear hepatocyte, $\mathbf{N}$ = nucleus. Coloration BMAll (Azur II-methylene blue). 

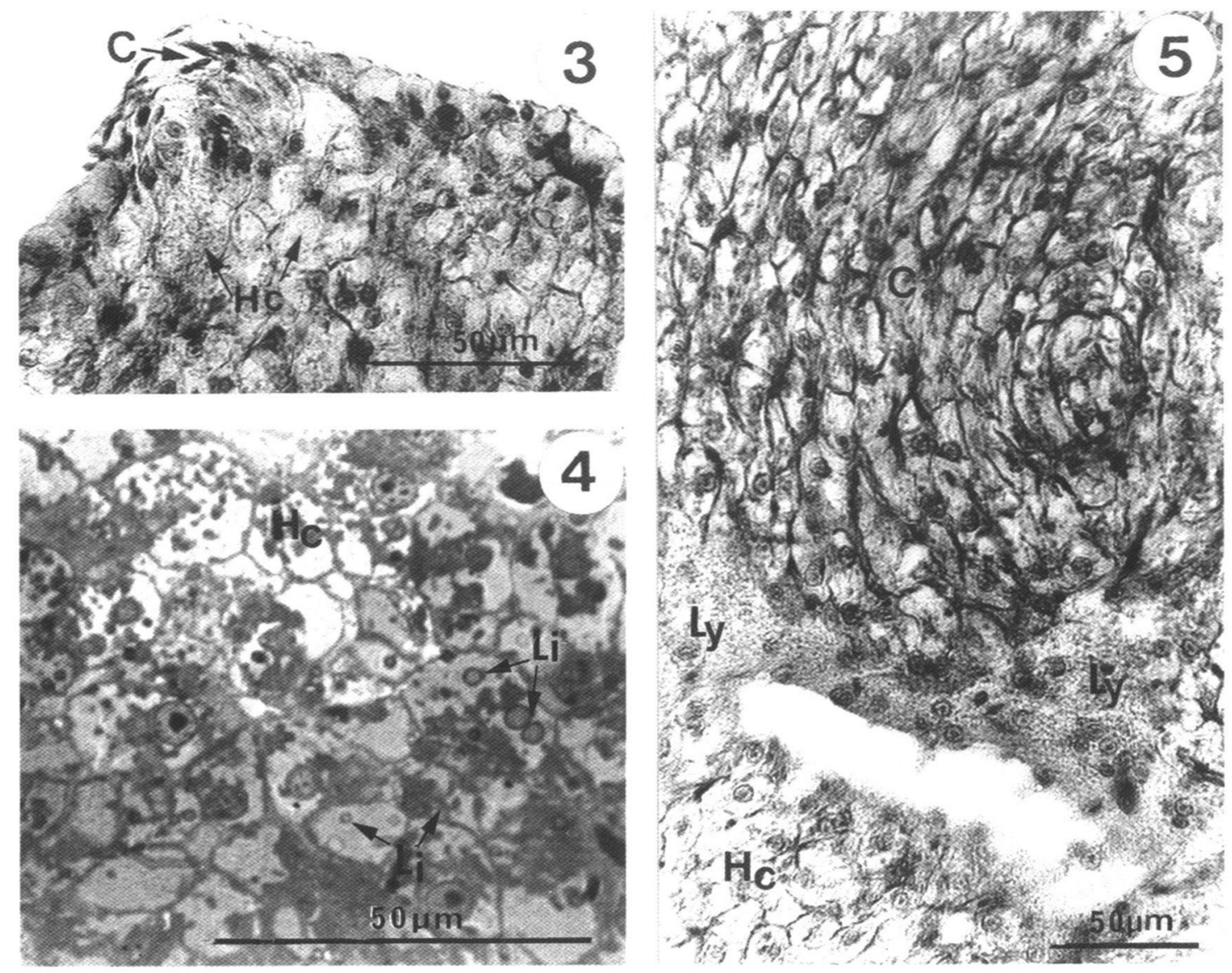

Figures 3 et 4

Après $14 \mathrm{j}$. de contamination, un arrangement concentrique des travées hépatocytaires $(\mathrm{C})$ est observé à proximité de foyers d'hépatocytes clairs $(\mathrm{Hc})$. Les hépatocytes proches des $\mathrm{Hc}$ contiennent de nombreuses inclusions lipidiques (Li). Coloration RNS-PIC.

\section{Figures 3 and 4}

After 14 days of exposure, concentric arrangement of hepatocytes (C) was observed near clear hepatocytes foci (Hc). In the vinicity of $\mathrm{Hc}$, hepatocytes contained numerous lipid inclusions (Li). Coloration RNS-PIC.

\section{Figure 5}

Après $14 \mathrm{j}$. de contamination suivis de $14 \mathrm{j}$. de décontamination, les organisations concentriques des travées hépatocytaires (C) de taille accrue se situent à proximité de plages de lyse (Ly) et de régions composées d'hépatocytes clairs (Hc). Coloration RNS-PIC.

\section{Figure 5}

After 14 days of exposure followed by 14 days of depuration, concentric patterns of hepatocytes (C) were large and located near lysis areas (Ly) and clear hepatocyte foci $(\mathrm{Hc})$. Coloration RNS-PIC. 

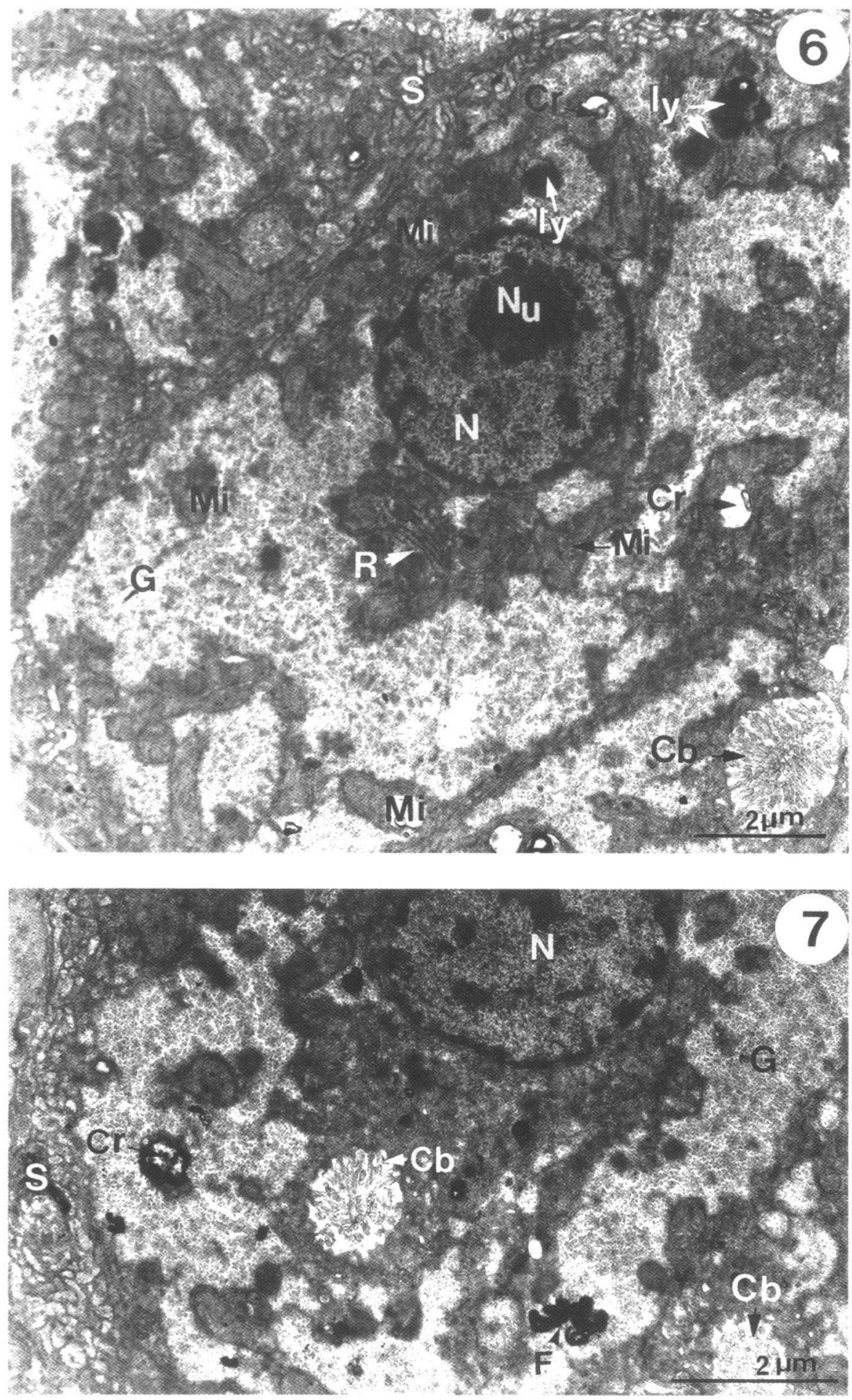

\section{Figures 6 et 7}

Organisation ultrastructurale des hépatocytes témoins. $\mathrm{Cb}=$ canalicule biliaire, $\mathrm{Cr}=$ corps résiduel, $\mathrm{G}=$ glycogène, $\mathrm{ly}=$ lysosome, $\mathrm{Mi}=$ mitochondrie, $\mathrm{N}=$ noyau, $\mathrm{Nu}=$ nucléole, $\mathrm{R}=$ réticulum endoplasmique granulaire, $\mathrm{S}=$ sinusoïde.

Figures 6 and 7

Ultrastructural organization of control hepatocytes. $\mathrm{Cb}=$ biliary canaliculus, $\mathrm{Cr}=$ residual body, $\mathrm{G}=$ glycogene, $\mathrm{ly}=$ lysosome, $\mathrm{Mi}=$ mitochondria, $\mathrm{N}=$ nucleus, $\mathrm{Nu}=$ nucleolus, $R=$ rough endoplasmic reticulum, $S=$ sinusoid. 
Les hépatocytes possèdent un noyau sphérique central et une bipolarité structurale et fonctionnelle ; ils présentent, en effet, un pôle basal biliaire où les canalicules biliaires sont bien visibles et un pôle apical périsinusoïdal pourvu de microvillosités (Figs. 6 et 7). Les canalicules biliaires, limités par des microvillosités formées par la membrane plasmique d'hépatocytes adjacents, pénètrent profondément à l'intérieur de ceux-ci jusqu'à proximité du noyau (Fig. 7). Les hépatocytes présentent, par ailleurs, une compartimentation cytoplasmique : les organites, mitochondries et réticulum endoplasmique granulaire (REG) en particulier, sont principalement localisés dans la région périnucléaire et péricellulaire. Le reste du cytoplasme est occupé par de vastes plages glycogéniques, les particules $\alpha$ de glycogène étant toutefois en nombre peu élevé chez ces femelles (Fig. 15). De rares globules lipidiques (non montrés sur les micrographies) de diamètre réduit (toujours inférieur à $0,5 \mu \mathrm{m}$ ) sont observables au sein des amas de glycogène. Quelques lysosomes secondaires, des corps résiduels et un nombre limité de figures myéliniques peuvent être détectés dans les régions péricanaliculaires. Ces structures lytiques participent au renouvellement normal des différents constituants cellulaires. Le noyau contient une hétérochromatine localisée essentiellement contre l'enveloppe nucléaire et un nucléole assez développé (Tableau II) dont les composantes fibrillaires et granulaires sont bien discernables (Fig. 6).

\section{Tableau II}

Paramètres cytométriques mesurés sur micrographies (15 mesures).

Table II

Cytometric parameters measured on micrographs (15 measurements).

\begin{tabular}{|c|c|c|c|}
\hline Individus & $\begin{array}{c}\text { Diamètre des noyaux } \\
(\mu \mathrm{m})\end{array}$ & $\begin{array}{c}\text { Diamètre des nucléoles } \\
(\mu \mathrm{m})\end{array}$ & $\begin{array}{c}\text { Taille des hépatocytes } \\
(\mu \mathrm{m})\end{array}$ \\
\hline Témoins & $4,8 \pm 1$ & $1,6 \pm 0,1$ & $15,9 \pm 2$ \\
\hline 4 j. d'intoxication & $4,2 \pm 1$ & $1,4 \pm 0,1$ & $18,0 \pm 2$ \\
\hline 7 j. d'intoxication & $4,3 \pm 1$ & $1,4 \pm 0,1$ & $13,2 \pm 2$ \\
\hline 14 j. d'intoxication & $3,7 \pm 2$ & $1,3 \pm 0,1$ & $10,0 \pm 2$ \\
\hline Décontamination & $4,2 \pm 1$ & $2,0 \pm 0,2$ & $15,5 \pm 2$ \\
\hline
\end{tabular}

\section{Perturbations (ultra)structurales du foie des danios contaminés}

\section{4 et 7 jours de contamination}

La procymidone provoque des perturbations hépatiques facilement observables par les techniques microscopiques. Dès 4 jours de contamination, l'altération la plus évidente est la lyse de quelques hépatocytes isolés ou de groupes d'hépatocytes, essentiellement localisés dans les régions périvasculaires (Fig. 2). L'endothélium est partiellement (Fig. 8) ou entièrement (Fig. 2) lysé, la structure typique des vaisseaux sanguins n'est, alors, plus observable. Après 7 jours, la dégénérescence s'aggrave, plus du quart du parenchyme hépatique est détruit et les importantes plages de lyse contiennent des débris cellulaires, en particulier des noyaux isolés (Fig. 2). Certains hépatocytes, plus volumineux que les autres (rapport nucléo-cytoplasmique réduit de $25 \%$ ) et présentant un cytoplasme clair, sont disséminés dans le parenchyme ou regroupés près des zones lysées. Les régions du parenchyme contenant ces hépatocytes sont déstructurées, puisque l'arrangement cellulaire en travées n'y est plus discernable (Fig. 2). 

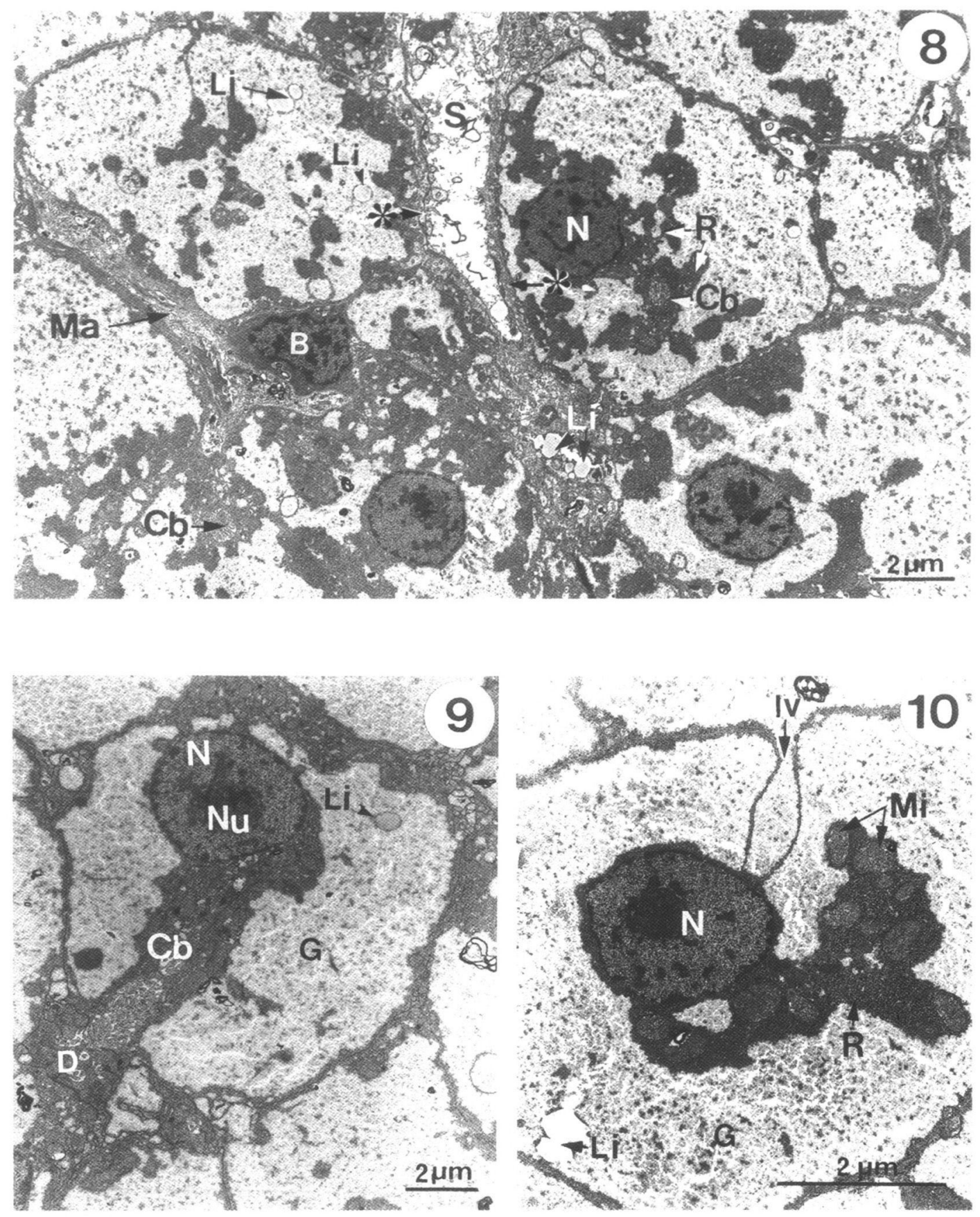

\section{Figure 8}

Après $4 \mathrm{j}$. d'intoxication, les hépatocytes sont pauvres en organites cytoplasmiques : mitochondries et réticulum $(R)$. Les microvillosités périsinusoïdales ont disparu $\left(^{*}\right)$. $\mathrm{B}=$ cellule biliaire, $\mathrm{Cb}=$ canalicule biliaire, $\mathrm{Li}=$ lipide, $\mathrm{Ma}=$ macrophage, $\mathrm{N}=$ noyau, $\mathrm{S}=$ sinusoïde.

\section{Figure 8}

After 4 days of exposure, hepatocytes contained few organelles : mitochondria and reticulum $(R)$. The perisinusoidal microvillies have disappeared $\left({ }^{\star}\right)$. $B=$ biliary cell, $\mathrm{Cb}=$ biliary canaliculus, $\mathrm{Li}=$ lipid, $\mathrm{Ma}=$ macrophage, $\mathrm{N}=$ nucleus, $\mathbf{S}=$ sinusoid. 


\section{Figure 9}

Après $7 \mathrm{j}$. de contamination, les hépatocytes montrent un pôle canaliculaire non altéré. $\mathrm{Cb}=$ canalicule biliaire, $\mathrm{D}=$ ductule biliaire, $\mathrm{G}=$ glycogène, $\mathbf{L i}=$ lipide, $\mathbf{N}=$ noyau, $\mathrm{Nu}=$ nucléole.

Figure 9

After 7 days of exposure, hepatocytes displayed non altered biliary canalicule $(\mathrm{Cb}) . \mathrm{D}=$ biliary ductule, $\mathrm{G}=$ glycogen, $\mathrm{Li}=$ lipid, $\mathbf{N}=$ nucleus, $\mathrm{Nu}=$ nucleolus.

\section{Figure 10}

Après $7 \mathrm{j}$. de contamination, des protrusions cytoplasmiques inter-hépatocytaires sont observables. Iv = invagination membranaire, $\mathbf{M i}=$ mitochondrie, $\mathbf{N}=$ noyau, $\mathbf{R}=$ réticulum endoplasmique granulaire.

\section{Figure 10}

After 7 days of exposure, cytoplasmic protrusions between adjacent hepatocytes were observed. Iv = membrane invagination, $\mathrm{Mi}=$ mitochondria, $\mathrm{N}=$ nucleus, $\mathbf{R}=$ rough endoplasmic reticulum.

Les hépatocytes (Figs. 9 et 10) sont très pauvres en organites cytoplasmiques (REG et mitochondries); quelques citernes du REG sont cependant toujours observables dans les régions périnucléaires et péricanaliculaires. La quantité des réserves glycogéniques varie d'un individu à l'autre, cependant les hépatocytes présentent généralement une diminution de leur charge glycogénique (Figs. 8 et 16). D'assez nombreux globules lipidiques (diamètre : $0,7 \pm 0,1 \mu \mathrm{m}$ ), entourés d'une membrane, sont observables au sein de ces plages glycogéniques; ils sont plus fréquents près des sinusoïdes ou à proximité des zones lysées (Fig. 8). Les microvillosités périsinusoïdales se nécrosent et disparaissent dès 4 jours d'exposition (Fig. 8). Par contre, les canalicules biliaires conservent un aspect semblable à celui des témoins (Fig. 9). Des protrusions cytoplasmiques d'un hépatocyte dans l'hépatocyte voisin (Fig. 10) sont très fréquentes, la membrane plasmique pénètre alors profondément dans le cytoplasme et parvient souvent au contact de l'enveloppe nucléaire. Ces invaginations sont toujours situées dans les régions latérales des cellules. D'assez nombreux macrophages sont présents dans le parenchyme où ils phagocytent les débris cellulaires et les cellules nécrosées ou lysées (Fig. 8).

Par ailleurs, une organisation particulière des travées hépatocytaires est révélée, en effet une disposition concentrique des hépatocytes autour de petites zones altérées est bien visible (Fig. 3). Ces structures concentriques sont fréquemment situées à proximité des régions lysées du parenchyme; si leur taille est le plus souvent réduite (environ $100 \mu \mathrm{m}$ ), elles peuvent atteindre des dimensions beaucoup plus importantes (plus de $900 \mu \mathrm{m}$ de diamètre). 

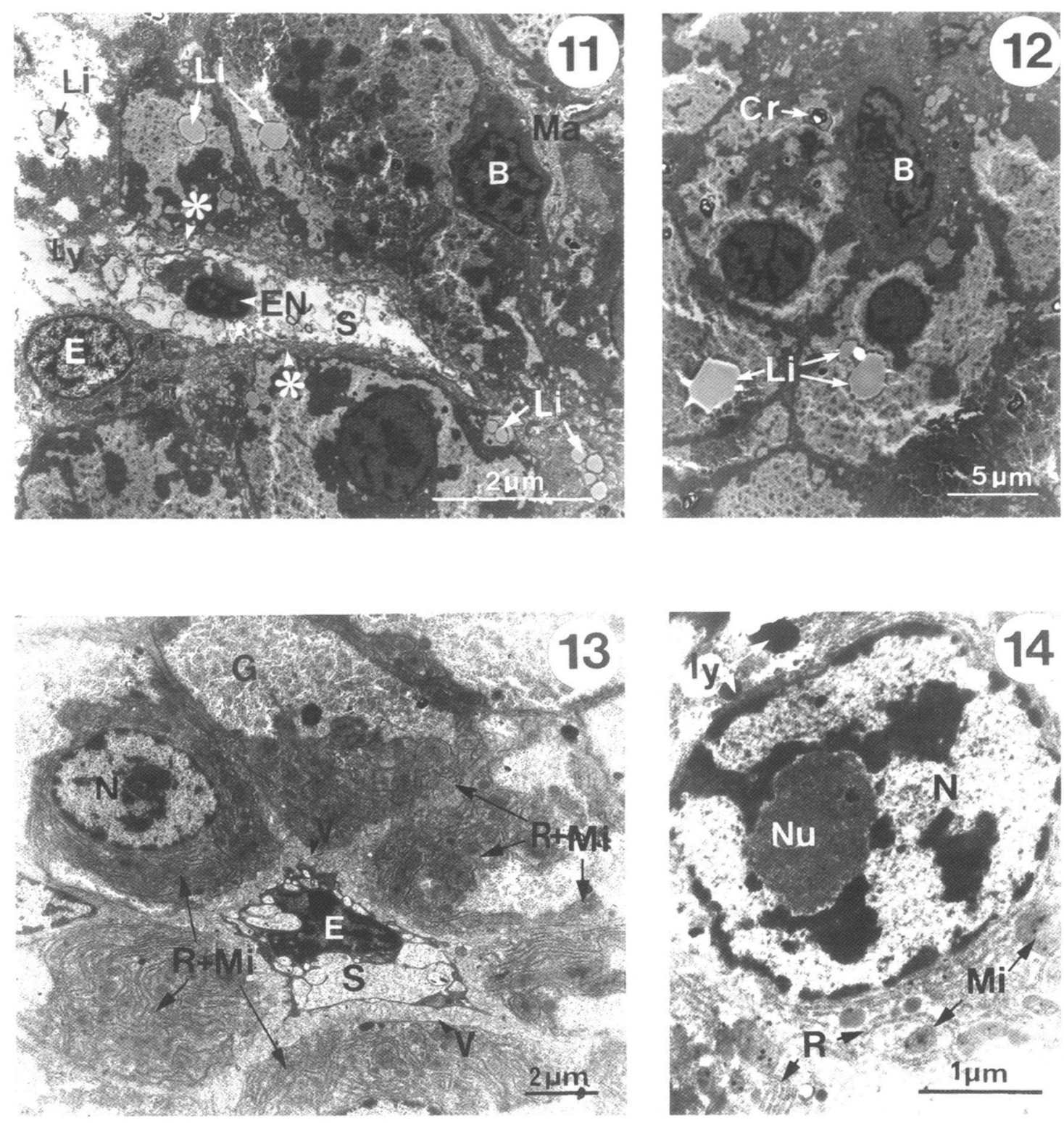

\section{Figures 11 et 12}

Après $14 \mathrm{j}$. de contamination, les hépatocytes présentent de nombreux globules lipidiques (Li), leurs microvillosités périsinusoïdales ont disparu (*). Les cellules endothéliales sont lysées (E) ou nécrosées (EN). $\mathrm{B}=$ cellule biliaire, $\mathrm{Cr}=$ corps résiduel, $L y=$ lyse, $M a=$ macrophage,$S=$ sinusoïde.

\section{Figures 11 and 12}

After 14 days of exposure, hepatocytes displayed numerous lipid inclusions and perisinusoidal microvillies have disappeared $\left(^{*}\right)$. Endothelial cells were lysed $(E)$ or necrotic $(\mathrm{EN}) . \mathrm{B}=$ biliary cell, $\mathrm{Cr}=$ residual body, $\mathrm{Ly}=$ lyse, $\mathrm{Ma}=$ macrophage, $\mathrm{S}=$ sinusoid. 
Figures 13 et 14

Après $14 \mathrm{j}$. de contamination suivis de $14 \mathrm{j}$. de décontamination ; Fig. 13 : un complexe très développé de réticulum endoplasmique granulaire associé aux mitochondries (R+Mi) occupe une part importante du cytoplasme. Les microvillosités périsinusoïdales $(V)$ sont restaurées. Fig. 14 : le nucléole $(\mathrm{Nu})$ a une taille importante. $E$ = cellule endothéliale, $G=$ glycogène, ly = lysosome, $\mathrm{Mi}=$ mitochondrie, $\mathbf{N}=$ noyau, $\mathbf{R}=$ réticulum endoplasmique granulaire, $\mathrm{S}=$ sinusoïde.

Figures 13 and 14

After 14 days of exposure followed by 14 days of depuration ; Fig. 13 : very developed rough endoplasmic reticulum associated to mitochondria $(R+M i)$ invaded important part of the cytoplasm. Perisinusoidal microvillies (V) were restored. Fig. 14 : the size of nucleolus $(\mathrm{Nu})$ has increased. $E$ = endothelial cell, $\mathbf{G}=$ glycogen, $\mathbf{l y}=$ lysosome, $\mathrm{Mi}=$ mitochondria, $\mathbf{N}=$ nucleus, $\mathbf{R}=$ rough endoplasmic reticulum, $\mathbf{S}=$ sinusoid.
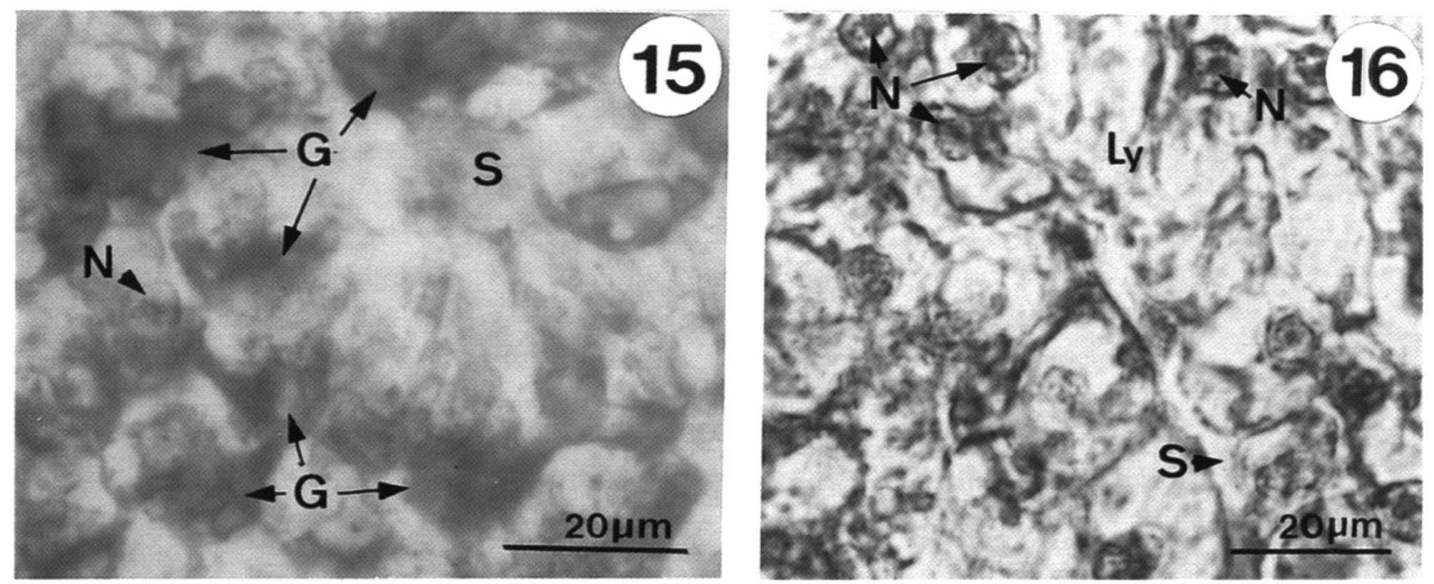

Figure 15

Parenchyme hépatique des danios témoins : les amas de glycogène, colorés par l'APS, occupent une importante part du cytoplasme des hépatocytes. $\mathbf{G}=$ glycogène, $\mathbf{N}=$ noyau, $\mathbf{S}=$ sinusoïde. Coloration APS.

\section{Figure 15}

Hepatic parenchyma of a control danio : hepatocyte cytoplasm contained large amount of glycogen stained by APS : periodic acid-Schiff. $G=$ glycogen, $\mathbf{N}=$ nucleus, $\mathbf{S}=$ sinusoid.

\section{Figure 16}

Après $7 \mathrm{j}$. de contamination, aucune présence de glycogène n'est révélée au sein des hépatocytes. $L y=$ lyse, $N=$ noyau, $S=$ sinusoïde. Coloration APS et hématoxiline de Groat.

\section{Figure 16}

After 7 days of contamination, no glycogen could be observed in hepatocytes. Ly = lyse, $\mathbf{N}=$ nucleus, $\mathbf{S}$ = sinusoid. Coloration APS and Groat's hematoxylin. 


\section{4 jours de contamination}

Près de la moitié du parenchyme hépatique est lysé et le quart du parenchyme restant est constitué par des hépatocytes volumineux, clairs et sans disposition en travées (Fig. 4). Lorsque les sinusoïdes sont encore reconnaissables, leurs cellules endothéliales sont très altérées (en voie de lyse ou de nécrose), et ils contiennent de nombreux débris cellulaires, en particulier des inclusions lipidiques de grande taille (Fig. 11). Les autres hépatocytes sont petits et présentent un nucléole de taille réduite (Tableau II). Les organites, mitochondries et réticulum, sont très rares dans le cytoplasme (Fig. 12). Les plages glycogéniques sont très pauvres en particules de glycogène (Figs. 11 et 12). Les globules lipidiques sont, par contre, nombreux et de taille importante (diamètre atteignant $3 \mu \mathrm{m}$ ), les hépatocytes clairs en sont particulièrement riches, à proximité des veinules ou des zones les plus altérées (Figs. 4 et 11). Un grand nombre de macrophages a été observé surtout dans les vaisseaux sanguins mais aussi dans les sinusoïdes proches des zones de lyse (Fig. 11). Les arrangements concentriques hépatocytaires de taille très variable (non montrés sur les micrographies) sont plus fréquents qu'après 7 jours d'intoxication.

\section{4 jours de contamination suivis de 14 jours de décontamination}

Les zones de lyses précédemment décrites sont moins nombreuses et remarquablement moins étendues (Fig. 5). Elles contiennent toujours des débris cellulaires divers mais surtout des érythrocytes et des macrophages en nombre important, ces derniers sont également nombreux dans le parenchyme et le système circulatoire. La réduction de taille des foyers d'hépatocytes clairs est aussi très nette (Fig. 5). Les arrangements concentriques des travées hépatocytaires demeurent, ils sont fréquemment de grandes dimensions (Fig. 5). De nombreux hépatocytes de taille normale contiennent toutefois peu de glycogène et quelques globules lipidiques (Tableau II, Fig. 13). Par contre, ils présentent un réticulum endoplasmique granulaire très développé associé à un nombre accru de mitochondries. Ces organites occupent près de la moitié du volume cytoplasmique et envahissent les zones normalement réservées au glycogène (Fig. 13). Le nucléole, dont les composants granulaire et fibrillaire sont discernables, présente une légère augmentation de taille (Tableau II, Fig. 14). Les microvillosités périsinusoïdales sont à nouveau bien visibles, elles retrouvent un aspect et une taille similaires à ceux des témoins (Fig. 13).

\section{DISCUSSION}

L'ultrastructure des hépatocytes des femelles de danio témoins correspond à celle précédemment décrite par PEUTE et al. (1978) et BRAUNBECK et al. (1989, 1990a); elle montre bien les spécificités ultrastructurales et fonctionnelles du foie des femelles en vitellogenèse mises en évidence par les études de ce dernier auteur concernant le dimorphisme sexuel hépatique. Les hépatocytes des femelles sont généralement de plus grande taille que ceux des mâles, ils possèdent un REG assez développé et bien organisé, leur charge glycogénique est nettement moins importante que celle des mâles. Les travaux réalisés chez les Poissons ont montré l'importance du sexe et du moment du cycle sexuel en ce qui concerne la réponse ultrastructurale du foie au stress induit par des xénobiotiques lipophiles (BRAUNBECK et al., 1989, 1990a). Notre étude a concerné les femelles en vitellogenèse car ces organismes pourraient montrer une sensibilité accrue aux toxiques. Par ailleurs, à ce moment, un transfert de polfuants du foie vers la gonade et leur incorporation dans les ovocytes au cours de la vitellogenèse exogène ovocytaire peuvent être suspectés (BRUSLE, 1982) et affecteraient ainsi la reproduction. 
L'exposition des danios à la concentration sublétale de $1 \mathrm{mg} . \mathrm{I}^{-1}$ de procymidone provoque une diminution des réserves glycogéniques hépatocytaires. Cette réponse bien connue au stress est non spécifique et a été induite après exposition de $B$. rerio à divers toxiques (GLUTH et HANKE, 1985 ; WRIGHT et al., 1989 ; BRAUNBECK et al., 1989, 1990a et b ; MOUTOU et al., 1997). Cette déplétion glycogénique du foie et l'hyperglycémie associée constituent des réponses à la demande énergétique accrue induite par un stress.

La contamination expérimentale de poissons d'espèces variées par des contaminants liposolubles de nature chimique très différente (hydrocarbures, polychlorobiphényles, contaminants industriels, pesticides...) provoque une accumulation rapide de lipides hépatiques (CORMIER et RACINE, 1990 ; revue de BIAGIANTIRISBOURG, 1997). Les lipides de réserve hépatocytaires constituent un site de "stockage" des substances toxiques liposolubles. Cette séquestration de molécules xénobiotiques à l'intérieur des globules lipidiques constituerait, selon STEGEMAN (1978) et BIAGIANTI-RISBOURG et BASTIDE (1995), un moyen de protection contre leurs effets toxiques. Toutefois, si au cours de l'intoxication par la procymidone un accroissement quantitatif des lipides a été observé, celui-ci s'accompagne d'une réduction de la concentration hépatique du fongicide. La bioaccumulation de toxiques constitue, selon GUNKEL (1981) et CLEVELAND et HAMILTON (1983), un mécanisme dynamique résultant des vitesses d'accumulation ("uptake») et de décontamination ("excretion"), un équilibre peut être atteint lorsque ces vitesses sont égales, le FB se stabilise alors tant que l'hépatocyte est physiologiquement fonctionnel. Ici, la disparition des microvillosités périsinusoïdales (réduction de la surface d'échange) constituerait une bioprotection cellulaire qui limiterait la pénétration du toxique. Après 4 jours d'exposition, la diminution du FB peut donc s'expliquer à la fois par la réduction de l'accumulation du fongicide et par le maintien de la décontamination hépatocellulaire. En effet, au cours de l'exposition à la procymidone, les canalicules biliaires ont toujours montré une ultrastructure identique à celle des témoins, il est possible que leur fonction d'excrétion du toxique ait été préservée.

L'accroissement quantitatif des lipides hépatocellulaires n'est donc pas corrélé à la quantité de procymidone bioaccumulée. L'accumulation lipidique proviendrait d'une inhibition de la synthèse des apoprotéines (réticulum endoplasmique déficient) impliquées dans le transfert des lipides de l'hépatocyte vers le compartiment extracellulaire et/ou d'une déficience du transport des vacuoles de condensation golgiennes par les microtubules (NOVIKOFF, 1982 ; HINTON et LAUREN, 1990). De plus, compte tenu de la réduction du nombre des mitochondries, une diminution du catabolisme des acides gras ( $($ oxydation) peut être mise en cause.

Nos observations révèlent, chez les danios contaminés, une réduction du nombre des citernes du réticulum endoplasmique. Cette réponse s'oppose à ce qui est généralement observé (hyperdéveloppement du réticulum) chez les Poissons exposés à de nombreux toxiques lipophiles et qui s'accompagne d'une induction du système MFO (SCHOOR et COUCH, 1979 ; STEGEMAN et KLOEPPER, 1987 ; NARBONNE et al., 1991). Il semble donc qu'il n'y ait pas stimulation de la protéosynthèse (enzymes de détoxication) et qu'un "blocage" du métabolisme hépatique ait lieu, celui-ci étant également révélé par la réduction du nombre des autres organites cytoplasmiques, mitochondries en particulier. Un tel blocage métabolique associé à la disparition des microvillosités périsinusoïdales a d'ailleurs précédemment été décrit chez Liza ramada intoxiquée par une concentration élevée d'atrazine (BIAGIANTI-RISBOURG, 1990).

L'exposition à $1 \mathrm{mg} . \mathrm{l}^{-1}$ de procymidone provoque des altérations sévères (lyse du parenchyme) qui montrent une dégénérescence importante de nombreux hépatocytes, cellules endothéliales et cellules biliaires. L'augmentation du nombre des macrophages au cours de l'intoxication correspond à une activation d'une réponse immunitaire cellulaire 
non spécifique qui peut être corrélée à l'accroissement de la destruction du tissu hépatique, tel que cela a été montré par STANTON (1965) et BRAUNBECK et al. (1989, 1990b).

Le retour en eau non contaminée provoque une réduction très nette de l'étendue et du nombre des zones lysées du parenchyme. Un développement du réticulum endoplasmique granulaire, une importante augmentation du nombre des mitochondries ainsi qu'un accroissement notable du diamètre des nucléoles sont autant de signes montrant une réactivation du métabolisme hépatocytaire (protéosynthèse accrue). Le rétablissement des échanges sang/hépatocyte est révélé par la restauration de la taille des microvillosités périsinusoïdales.

Au cours de l'exposition à la procymidone et de la décontamination, le développement d'arrangements concentriques des travées hépatocytaires est observable. La signification de l'apparition et du maintien de telles structures, a priori dégénératives, n'est pas encore connue.

La protrusion de portions cytoplasmiques d'un hépatocyte à l'intérieur de l'hépatocyte voisin constitue une perturbation provoquée au cours de la contamination, mais qui disparaît après 14 jours de décontamination. Elle permet un contact inhabituel entre les membranes plasmiques et l'enveloppe nucléaire. De telles invaginations de la membrane plasmique constituent une réponse originale provoquée par le fongicide. L'explication de cette perturbation, qui augmente la surface de l'hépatocyte, est également encore inconnue.

\section{CONCLUSION}

Ce travail qui s'inscrit dans le cadre d'études de toxicologie de l'environnement, montre clairement un impact toxique de la procymidone au niveau hépatique chez le Cyprinidé $B$. rerio. Cependant, la concentration en fongicide utilisée ici est nettement supérieure à celles rencontrées dans les milieux aquatiques contaminés (de l'ordre d'une dizaine de microgrammes). Cette concentration hépatotoxique proche des doses létales a été choisie dans le but de mieux comprendre les mécanismes dégénératifs induits par ce fongicide et de mettre en évidence certains biomarqueurs (ultra)structuraux de pollution, ce qui constitue une orientation de recherches actuelle privilégiée (HINTON et al., 1992 ; BRAUNBECK, 1995 ; BIAGIANTI-RISBOURG, 1997). Elle a permis de montrer que, lors d'une contamination sévère, si les atteintes dégénératives sont importantes (après $14 \mathrm{j}$. d'exposition, près de la moitié du parenchyme hépatique est lysée) les autres hépatocytes toujours fonctionnels développent des mécanismes de protection (disparition des microvillosités, accumulation lipidique) qui permettent probablement la survie du poisson.

\section{BIBLIOGRAPHIE}

AFNOR 85202, norme française NF T 90-303, juin 1985.

BIAGIANTI-RISBOURG S., 1990. Contribution à l'étude du foie de juvéniles de muges (Téléostéens, Mugilidés) contaminés expérimentalement par l'atrazine (s-triazine herbicide) : Approche ultrastructurale et métabolique ; Intérêt en écotoxicologie. Thèse doctorat d'Etat, Univ. de Perpignan, 1-451.

BIAGIANTI-RISBOURG S., 1997. Les perturbations (ultra)structurales du foie des poissons utilisées comme biomarqueurs de la qualité sanitaire des milieux aquatiques. In Biomarqueurs en Ecotoxicologie - Aspects Fondamentaux, LAGADIC L., CAQUET T., AMIARD J.C. et RAMADE F. (eds.), Masson, Paris, 355-391. 
BIAGIANTI-RISBOURG S. and BASTIDE J., 1995. Hepatic perturbations induced by a herbicide (atrazine) in juvenile grey mullet Liza ramada (Mugilidae, Teleostei) : an ultrastructural study. Aquatic Toxicol., 31, 3, 217-229.

BIAGIANTI-RISBOURG S., PAIRAULT C., VERNET G. and BOULEKBACHE H., 1996. Effect of Lindane on the ultrastructure of the liver of the rainbow trout, Oncorhynchus Mykiss, sac-fry. Chemosphere, 33, 10, 2065-2079.

BRAUNBECK T., 1995. Detection of environmentally relevant concentrations of toxic organic compounds using histological and cytological parameters : substancespecificity in the reaction of rainbow trout liver? Proceed. EIFAC Symp. "Sublethal and chronic effects of pollutants on freshwater fish", Lugano, May 1992, 1-14.

BRAUNBECK T., STORCH V. and NAGEL R., 1989. Sex-specific reaction of liver ultrastructure in zebrafish (Brachydanio rerio) after prolonged sublethal exposure to 4-nitrophenol. Aquatic Toxicol., 14, 185-202.

BRAUNBECK T., GORGE G., STORCH V. and NAGEL R., 1990a. Hepatic steatosis in zebrafish (Brachydanio rerio) induced by long-term exposure to a-hexachlorohexane. Ecotoxicol. Environ. Safety, 19, 355-374.

BRAUNBECK T., STORCH V. and BRESCH H., 1990b. Species-specific reaction of liver ultrastructure in zebrafish (Brachydanio rerio) and trout (Salmo gairdnerl) after prolonged exposure to 4-chloroaniline. Arch. Environ. Contam. Toxicol., 19, 405418.

BRESCH H., 1993. Some remarks to a long-term toxicity test in fish for ecological purposes. In Fish Ecotoxicology and Ecophysiology, BRAUNBECK T., HANKE W., SEGNER H. (eds.), VCH, Weinheim, New York, Basel, Cambridge, 127-132.

BRUSLE S., 1982. Contribution à la connaissance de la sexualité de poissons Téléostéens marins gonochoriques (Mugilidés) et hermaphrodites (Serranidés). Thèse doctorat d'Etat, Univ. de Perpignan, 1-360.

CLEVELAND L. and HAMILTON S.J., 1983. Toxicity of the organophosphorus defoliant DEF to rainbow trout (Salmo gairdneri) and channel catfish (/ctalurus punctatus). Aquatic Toxicol., 4, 341-355.

CORMIER S.M. and RACINE R.N., 1990. Histopathology of atlantic tomcod : a possible monitor of xenobiotics in Northeast tidal rivers and estuaries. In Biomarkers of environmental contamination, McCARTHY J.F., SHUGART L.R. (eds.), Lewis Pubs., CRC Press, Boca Raton USA, 59-71.

GLUTH G. and HANKE W., 1985. A comparison of physiological changes in carp, Cyprinus carpio, induced by several pollutants at sublethal concentrations. The dependency on exposure time. Ecotoxicol. Environ. Saf., 9, 179-188.

GUNKEL G., 1981. Bioaccumulation of a herbicide (atrazine, s-triazine) in the whitefish (Coregonus fera J.) : uptake and distribution of the residue in fish. Arch. Hydrobiol. Suppl., 59, 252-287.

HINTON D.E. and LAUREN D.J., 1990. Liver structural alterations accompanying chronic toxicity in fishes : potential biomarkers of exposure. In Biomarkers of environmental contamination, McCARTHY J.F., SHUGART L.R. (eds.), Lewis Publishers, CRC Press, Boca Raton USA, 17-57.

HINTON D.E., BAUMANN P.C., GARDNER G.R., HAWKINS W.E., HENDRICKS D.J., MURCHELANO R.A. and OKIHIRO M.S., 1992. Histopathologic biomarkers. In Biomarkers, Biochemical, Physiological and Histological Markers of Anthropogenic Stress, HUGGETT R.J., KIMERLE R.A., MEHRLE P.M. and BERGMAN H.L. (eds.), Lewis Publishers, Boca Raton, Ann Arbor, London, Tokyo, 155-209.

LAALE H.W., 1977. The biology and use of zebrafish, Brachydanio rerio, in fisheries research. A literature review. J. Fish Biol., 10, 121-173. 
MARTOJA R. et MARTOJA-PIERSON M., 1967. Initiation aux techniques de l'histologie animale. Masson (ed.), Paris.

MOUTOU K.A., BRAUNBECK T. and HOULIHAN D.F., 1997. Quantitative analysis of alterations in liver ultrastructure of rainbow trout Oncorhynchus mykiss after administration of the aquaculture antibacterials oxolinic acid and flumequine. Dis. Aquat. Org., 29, 21-34.

NAGEL R., 1993. Fish and environmental chemicals - a critical evaluation of tests. In Fish Ecotoxicology and Ecophysiology, BRAUNBECK T., HANKE W., SEGNER H. (eds.), $\mathrm{VCH}$, Weinheim, New York, Basel, Cambridge, 147-156.

NARBONNE J.F., RIBERA D., MICHEL X., RAOUX C., GARRIGUES P., MONOD J.L., LEMAIRE P., GALGANI F., ROMEO M., SALAUN J.P. et LAFAURIE M., 1991. Indicateurs biochimiques de contamination de l'environnement marin : étude comparative en mer Méditerranée. Océanis, 17, 257-275.

NOVIKOFF A.B., 1982. Intracellular organelles and lipoprotein metabolism in normal and fatty livers. In The liver Biology and Pathobiology, ARIAS I.M., POPPER H., SHACHTER D., SHAFRITZ D.A. (eds.), Raven press N.Y., 143-167.

OULMI Y., NEGELE R.D. and BRAUNBECK T., 1995. Cytopathology of liver in rainbow trout Oncorhynchus mykiss after long-term exposure to sublethal concentrations of linuron. Dis. Aquat. Org., 21, 35-52.

PEUTE J., VAN DER GAAG M.A. and LAMBERT J.C.D., 1978. Ultrastructure and lipid content of the liver of Zebrafish, Brachydanio rerio, related to vitellogenin synthesis. Cell Tiss. Res., 186, 297-308.

PHROMKUNTHONG W., STORCH V. and BRAUNBECK T., 1994. Sexual dimorphism in the reaction of zebrafish (Brachydanio rerio) to ascorbic acid deficiency : induction of steatosis in hepatocytes of male fish. J. Appl. Ichtyol., 10, 146.

REYNOLDS E.S., 1963. The use of lead citrate at high $\mathrm{pH}$ as an electron opaque stain in electron microscopy. J. Biophys. Cytol., 17, 208-212.

RICHARDSON K.C., JARRET L. and FINKE E.H., 1960. Embedding in epoxy resin for ultrathin sectionning in electron microscopy. Stain Technol., 35, 313-323.

SCHOOR W.P. and COUCH J.A., 1979. Correlation of mixed-function oxidase activity with ultrastructural changes in the liver of a marine fish. Cancer Biochem. Biophys., 4, 95-103.

STANTON M.F., 1965. Diethylnitrosamine-Induced Hepatic Degeneration and neoplasia in the aquarium fish, Brachydanio rerio. J. Nat. Cancer Inst., 34, 117-130.

STEGEMAN J.J., 1978. Fate and effects of oil in marine animals. Océanis, 20, 4, 59-66.

STEGEMAN J.J. and KLOEPPER P.J., 1987. Cytochrome P-450 isozymes and monooxygenase activity in aquatic animals. Environ. Health Perspect., 17, 87-95.

WRIGHT P.A., PERRY S.F. and MOON T.W., 1989. Regulation of hepatic glucogenesis and glycogenolysis by catecholamines in rainbow trout during environmental hypoxia. J. exp. Biol., 147, 169-188. 